\title{
Digital Modulation and Coding for Satellite Optical Feeder Links with Pre-distortion Adaptive Optics
}

\author{
Svilen Dimitrov*, Ricardo Barrios, Balazs Matuz, Gianluigi Liva, Ramon Mata-Calvo, \\ Dirk Giggenbach
}

German Aerospace Center (DLR), Satellite Networks Department, 82234 Wessling, Germany

\begin{abstract}
SUMMARY
In this paper, a packet-level forward error correction (FEC) coding technique and pre-distortion adaptive optics (AO) technology are applied to a digital transmission scheme for optical feeder links in a geostationary Earth orbit (GEO) satellite communication system. The architectures of the gateway and the satellite are defined, including the building blocks of the interface between the radio frequency (RF) front-end and the optical front-end, as well as the digital signal processor. The system is designed to cater for Terabit/s highthroughput satellite (HTS) applications. The performance of the digital transmission scheme is evaluated in the forward and return links. The turbulent atmospheric optical channel is modeled for different optical ground station (OGS) altitudes. It is shown that fade mitigation techniques such as packet-level FEC coding and pre-distortion $\mathrm{AO}$ in the forward link, as well as large-aperture OGS telescope in the return link, are essential to close the link budget of a Terabit/s satellite communication system. Copyright (c) 2015 John Wiley \& Sons, Ltd.
\end{abstract}

Received ...

KEY WORDS: Terabit/s satellite communication; optical feeder link; pre-distortion adaptive optics; digital modulation; packet-level coding; scintillation; correlated fading; turbulent optical channel.

\section{INTRODUCTION}

The traffic demand for satellite broadband is expected to grow 6 -fold by 2020 [1]. To serve this increasing demand, next generation satellites need to offer both higher throughput and higher data rates, while decreasing the cost per transmitted bit. Terabit/s satellite communication systems are studied in the European Project on Broadband Access via Integrated Terrestrial and Satellite Systems (BATS) [1]. One key technology to transport large traffic to the Ka-band user link is the use of free space optical (FSO) feeder links [2]. This solution offers significantly larger bandwidth in the optical domain as compared to RF gateways in $\mathrm{Q} / \mathrm{V}$ band which leads to a reduced number of feeder links and facilitates centralized interference management.

\footnotetext{
${ }^{*}$ Correspondence to: German Aerospace Center (DLR), Satellite Networks Department, 82234 Wessling, Germany. Email: svilen.dimitrovedlr.de 
Index-of-refraction turbulence (IRT) [3] is known to degrade the achievable data rates of FSO communication links [4]. For uplinks above $30^{\circ}$ elevation, the IRT falls within the weak turbulence regime [5], where the probability density function (PDF) of the irradiance at the receiver plane can be modeled by a log-normal distribution [6]. Moreover, the correlated fading events represent a lowpass process defined by the Greenwood frequency [7]. Regarding site selection and deployment, an OGS at sea level may suffer from strong influence of the turbulence, since the atmosphere is denser at lower altitudes. Astronomical sites are generally located on mountain tops, but these locations are usually isolated, and Terabit/s infrastructure needs to be developed. In this paper, $300 \mathrm{~m}, 900 \mathrm{~m}$ and $1500 \mathrm{~m}$ are chosen as examples of possible OGS altitudes.

Adaptive optics (AO) is a technology used for correction of the incoming optical wavefront phase which is distorted by the turbulent atmosphere [8]. In ground-to-GEO FSO links, the scale size of the phase distortions is much larger than the feasible satellite telescope aperture size [3], and therefore, the spatial phase at the satellite receiving aperture is rather flat. Nevertheless, in an uplink scenario the traveling wavefront is initially perturbed by the atmosphere, and then it propagates through free space up to the GEO satellite. Thus, at the satellite plane the received wavefront profile differs from that of a pure diffracted laser beam. These distortions can be mitigated by applying pre-distortion AO (PAO) techniques to the outgoing optical wave at the ground station, using a beacon reference that senses the distortions introduced by the turbulent atmosphere.

The simplest PAO correction method is tilt compensation of the first three spatial modes of the wavefront phase information [9]. It uses pointing by tracking, where a downlink beacon from the satellite is used as a reference to point the outgoing beam. This method only minimizes the beam wander in the satellite plane. Additional gain can be obtained, if higher modes of the phase are also pre-compensated, since the higher modes are responsible for the phase distortions that mainly contribute to the IRT-induced scintillation effect. In this work, the performance of PAO is analyzed, when both lower and higher spatial modes are pre-compensated.

In general, return-to-zero (RZ) and non-return-to-zero (NRZ) modulation formats are widely used in optical data networks with intensity modulation and direct detection (IM/DD) [10]. While RZ modulation is more power efficient as compared to NRZ modulation, it requires twice the bandwidth to achieve a target throughput. These techniques are commonly used in conjunction with differential phase shift keying (DPSK) or on-off keying (OOK) [11]. Even though DPSK transmission shows an improved power efficiency as compared to OOK, it is significantly impaired in the presence of non-linear phase noise over long distances, and it has a lower spectral efficiency. Therefore, in this study, NRZ-OOK is chosen for application in HTS optical feeder links.

High data rates over a fading channel can be successfully achieved, provided that robust recovery mechanisms are implemented. Capacity approaching FEC techniques [12] such as low-density parity-check (LDPC) codes and turbo codes are at the heart of the latest standards for satellite communications, the digital video broadcasting via satellite 2 extensions (DVB-S2X) [13] and the DVB return channel via satellite 2 (DVB-RCS2) [14] standards for forward and return links, respectively. Fade durations in the order of tens of milliseconds can be compensated by a careful choice of the coding scheme. In order to limit the complexity, the redundancy can be split over twostage codes: an inner code is intended to correct (sporadic) bit errors due to channel noise, while an outer code is meant to compensate packet losses due to (long) fading events. After decoding of the inner code, each codeword (a symbol of the outer code) must pass an integrity check, for instance, 
in form of a cyclic redundancy check (CRC). As a result, it is possible to mark each symbol of the packet-level code either as correct or corrupted (erased). Next, the packet-level decoder attempts to recover the erased symbols. Since the packet-level code can span over thousands of symbols, each symbol being a packet of thousands of bits, sufficient time diversity to counteract long channel impairments can be provided.

In this paper, a digital transmission scheme based on NRZ-OOK is proposed for satellite optical feeder links. In addition, a packet-level FEC coding scheme is designed to counteract the IRT in the atmospheric optical channel in the forward link, as well as pre-distortion AO techniques are applied at the transmitter side. The gateway and satellite architectures are defined, including the RF-optical interface and the digital signal processor, to support Terabit/s throughput over Ka-band user beams. The atmospheric turbulent channel is modeled for $55^{\circ}$ elevation setups at $300 \mathrm{~m}, 900 \mathrm{~m}$ and $1500 \mathrm{~m}$ OGS altitudes. The performance of the digital transmission scheme is evaluated in this channel setup. Pre-distortion AO techniques reduce the scintillation index and the total link loss in the turbulent atmospheric optical channel in the forward link, while the application of packet-level FEC coding enables the system to close the link budget, i.e. meet the error rate target for the received signal power. The return link is shown to be less of a challenge due to the employed large-aperture OGS receive telescope.

The rest of the paper is organized as follows. Section 2 describes the atmospheric optical channel model. Section 3 introduces the pre-distortion AO model, and Section 4 elaborates on the performance evaluation scenarios. Section 5 presents the digital transmission scheme architecture. Section 6 introduces the packet-level coding scheme for the forward link, while Section 7 presents the performance evaluation results. Finally, Section 8 concludes the paper.

\section{ATMOSPHERIC OPTICAL CHANNEL}

The main impairments in the atmospheric optical channel are the IRT and beam wander. In this section, statistical models for these phenomena are presented, as well as the conditions for the application of pre-distortion AO techniques are elaborated.

\subsection{Refractive index structure}

The atmospheric turbulence can be characterized by the strength of the refractive index fluctuations represented by the refractive index structure parameter $C_{n}^{2}$ in units of $\left[\mathrm{m}^{-2 / 3}\right]$. When a vertical path is considered, as in a satellite feeder link scenario, the behavior of $C_{n}^{2}$ is mainly affected by temperature changes along the different layers within the Earth's atmosphere. Therefore, the refractive index structure parameter becomes a function of the height $h$ above the ground level. The most widespread $C_{n}^{2}$ profile model is the Hufnagel-Valley (HV), best suited for inland daytime and nighttime conditions. In this work, a modified version of the HV model is used, in order to include the ground layer atmospheric effects on an OGS not located at sea level [15]. Thus, the vertical profile of the refractive index structure parameter is expressed as follows:

$$
C_{n}^{2}(h)=A e^{-H_{\mathrm{GS}} / 700} e^{-\left(h-H_{\mathrm{GS}}\right) / 100}+5.94 \times 10^{-53}\left(\frac{v}{27}\right)^{2} h^{10} e^{-h / 1000}+2.7 \times 10^{-16} e^{-h / 1500},
$$




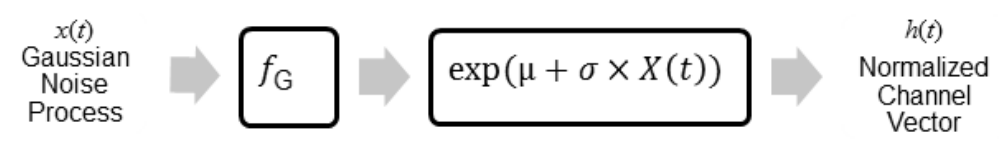

Figure 1. Block diagram of correlated time series synthesizer.

where $A=10^{-13}$ is the refractive index structure parameter at ground level in $\left[\mathrm{m}^{-2 / 3}\right], v=21$ is the root-mean-squared (RMS) wind speed in $\left[\mathrm{m} \mathrm{s}^{-1}\right]$, which are the standard values for the $\mathrm{HV} C_{n}^{2}$ model for daytime conditions, and $H_{\mathrm{GS}}$ is the optical ground station altitude.

\subsection{Scintillation index and Greenwood frequency}

In an FSO link, after the optical wave propagates through the turbulent atmosphere, the intensity seen by the receiver is a random variable. The scintillation index (SI) is used to quantify the strength of these irradiance fluctuations. In the uplink channel, the SI is defined as [3]:

$$
\sigma_{I}^{2}=\exp \left[\frac{0.49 \sigma_{I_{\mathrm{Bu}}}^{2}}{\left(1+0.56(1+\Theta) \sigma_{I_{\mathrm{Bu}}}^{12 / 5}\right)^{7 / 6}}+\frac{0.51 \sigma_{I_{\mathrm{Bu}}}^{2}}{\left(1+0.69 \sigma_{I_{\mathrm{Bu}}}^{12 / 5}\right)^{5 / 6}}\right]-1,
$$

where $\Theta$ is a parameter describing the beam phase front radius at the receiver plane, and $\sigma_{I_{\mathrm{Bu}}}^{2}$ is the uplink Rytov variance for Gaussian beams, which depends directly on the selected $C_{n}^{2}$ profile model and its corresponding expressions can be found in [3, Ch. 12].

The SI parameter represents the variation of the incoming signal in terms of ensemble averages, but it does not provide any information on the temporal behavior. In general, the IRT can be regarded as a low-pass process with a certain cut-off frequency, i.e. the Greenwood frequency, given by [7]:

$$
\begin{gathered}
f_{\mathrm{G}}=2.31 \lambda^{-6 / 5}\left[\int C_{n}^{2}(z) V^{5 / 3}(z) d z\right]^{3 / 5}, \\
V(h)=\omega_{\mathrm{s}} h+V_{\mathrm{g}}+30 \exp \left[-\left(\frac{h-9400}{4800}\right)^{2}\right],
\end{gathered}
$$

where $V(z)$ is the wind velocity profile along the propagation path $z=\sec (\zeta)\left(h-H_{\mathrm{GS}}\right), \omega_{\mathrm{s}}=$ $7.3 \times 10^{-5} \mathrm{rad} \mathrm{s}^{-1}$ is the GEO satellite slew rate, and $V_{\mathrm{g}}=8 \mathrm{~m} \mathrm{~s}^{-1}$ is the wind speed at ground level.

As a result, the channel in an optical feeder link scenario is completely characterized in terms of the statistical behavior of the received optical intensity at the GEO satellite through the SI, and in terms of the temporal behavior by means of the Greenwood frequency. Here, only weak-turbulence scenarios are considered and, thus, the PDF of the irradiance can be modeled by a log-normal distribution [6]. It takes a mean value $\mu$ and variance $\sigma^{2}$ as scale and shape parameters, respectively. These parameters are related to the SI as follows: $\mu=-\sigma^{2} / 2$ and $\sigma^{2}=\ln \left(\sigma_{I}^{2}+1\right)$, where $\sigma_{I}^{2}$ is defined in (2). A process for the generation of correlated time series according to these distributions by means of a low-pass filter is shown in Figure 1 [16]. 


\subsection{Beam wander}

The beam wander, $\left\langle r_{c}^{2}\right\rangle$, measures the average displacement of the beam at the receiver from the boresight. It is well known that this phenomenon is caused by the large-scale random inhomogeneities due to their refractive effects. Thus, the beam center can exhibit major deviations from the link optical axis after propagating through the turbulent atmosphere. The integrated turbulence strength along the optical path through the atmosphere can be measured by means of the Fried parameter $r_{0}$, where smaller values correspond to stronger atmospheric IRT conditions. The Fried parameter is given by [17]:

$$
r_{0}=\left[0.42 \sec (\zeta) k^{2} \int_{H_{\mathrm{GS}}}^{H} C_{n}^{2}(h) d h\right]^{-3 / 5},
$$

where $\zeta$ is the zenith angle in radians, $k=2 \pi / \lambda$ is the optical wavenumber, $\lambda$ being the wavelength, and $H$ is the satellite altitude. Therefore, the beam wander can be calculated as follows:

$$
\left\langle r_{c}^{2}\right\rangle=0.5\left(H-H_{\mathrm{GS}}\right)^{2} \sec (\zeta)\left(\frac{\lambda}{2 W_{0}}\right)^{2}\left(\frac{2 W_{0}}{r_{0}}\right)^{5 / 3},
$$

where $W_{0}$ is the beam radius at the transmitter plane.

\subsection{Conditions for pre-distortion}

The turbulence induced beam wander can be compensated by tracking the angle of arrival (AoA) of a reference signal from the satellite and pointing the outgoing beam accordingly. In addition, the wavefront modes at the receiver can be pre-distorted by means of AO technology applied at the transmitter. This method only works when the up- and downlink optical beams propagate through the same volume of atmosphere, since the downlink signal is used as a reference for correction of the uplink signal. This, however, is only applicable when the point-ahead angle (PAA), which is typically about $18 \mu \mathrm{rad}$, is smaller than the isoplanatic angle (IPA). The former is the angular separation between the down- and uplink signal, owing to the relative velocity of the satellite with respect to the OGS, while the latter defines the angular size where the atmospheric distortions can be considered constant. A graphical representation of the relationship between the PAA and the IPA is shown in Figure 2. Two scenarios are illustrated, one for high elevation angle and one for low elevation angle. The satellite moves approximately $700 \mathrm{~m}$ during the time of flight of down- and uplink, resulting in a required PAA of $18 \mu \mathrm{rad}$. The atmospheres IPA can be larger than the PAA at high link elevations, or smaller at low elevations. For beam tracking and pre-distortion the following condition should hold: IPA > PAA. The IPA can be calculated as follows [3]:

$$
\mathrm{IPA}=\cos ^{8 / 5}(\zeta)\left[2.91 k^{2} \int_{H_{\mathrm{GS}}}^{H} C_{n}^{2}(h)\left(h-H_{\mathrm{GS}}\right)^{5 / 3} d h\right]^{-3 / 5}
$$

In Figure 3 the isoplanatic angle is plotted as a function of the elevation angle for three OGS altitudes, namely 300,900 and $1500 \mathrm{~m}$. Therefore, an elevation angle of $55^{\circ}$ is chosen for the study on improvement of the total link loss, including improvement of the Fried parameter and beam 


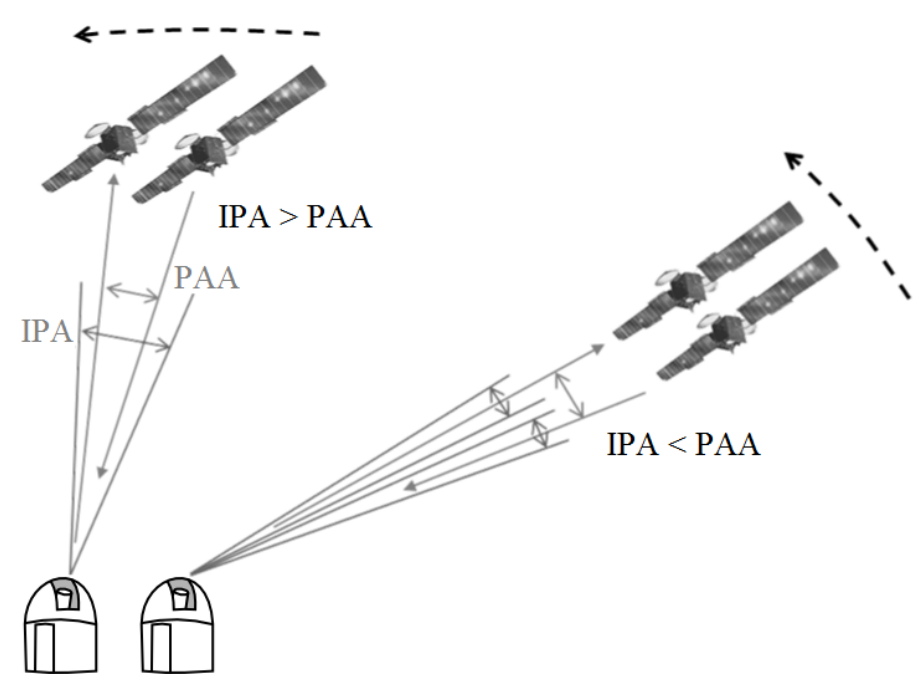

Figure 2. Scenarios for an optical GEO feeder link, illustrating the satellite movement during the time of flight of down- and uplink, as well as the relation between IPA and PAA at high and low elevation angles.

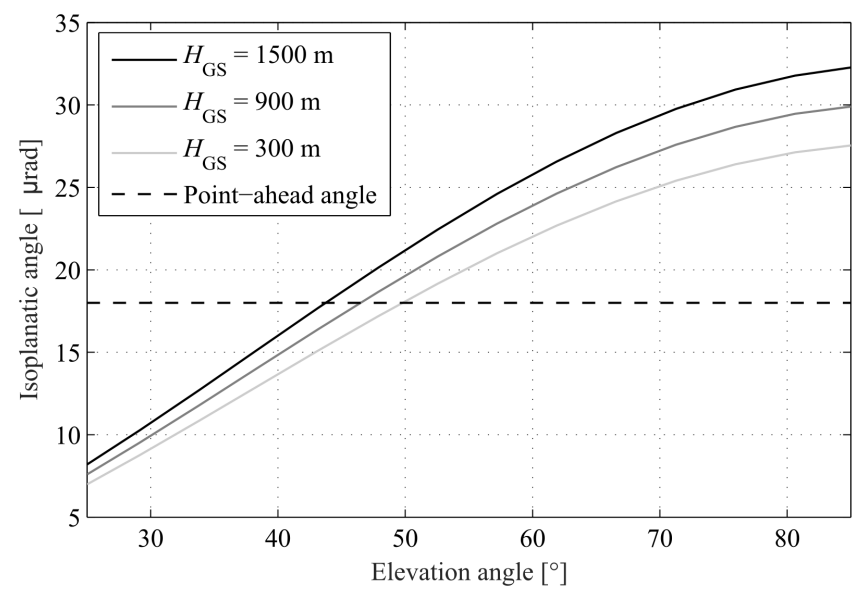

Figure 3. Isoplanatic angle $v s$. elevation angle for ground station altitudes of $900 \mathrm{~m}$ and $1500 \mathrm{~m}$.

wander, as well as the scintillation index, through the application of pre-distortion AO technology in this paper.

\section{PRE-DISTORTION ADAPTIVE OPTICS}

Optical wave propagation through the turbulent atmosphere produces self-interference on the traveling wave. This translates into phase distortions that are responsible for the stochastic degradation of the transmitted energy. Traditionally, the phase of an optical wave passing through a circular aperture is represented by an infinite summation of modes commonly described by the orthogonal basis given by the Zernike polynomials [9]. Each Zernike mode is associated with a Zernike coefficient which can be efficiently estimated using algorithms to solve linear 
systems $[18,19,20]$. These sets of polynomials are the most convenient representation for the optical wave phase, as they can be related to classical aberrations in optical systems. For instance, the first Zernike mode represents the averaging effect of a circular receiving aperture. The next two modes represent the vertical and horizontal tilt of the incoming wavefront which together give the AoA that can be used to correct for beam wander effects in the uplink. On one hand, correcting the tilt components of the optical wave suppresses most of the deep fades in the uplink. On the other hand, higher modes, i.e. higher spatial frequencies, are responsible for scintillation effects that can only be corrected applying pre-distortion to the uplink laser beam.

A PAO system consists basically of four main elements, namely a wavefront sensor, wavefront processor, laser source and conjugation element. The wavefront measuring device, usually a ShackHartmann sensor, is used together with a corresponding processor to effectively estimate the conjugated phase needed to be applied to an initially flat wavefront laser source by means of a conjugation element. The phase conjugation operation is normally executed in two stages: first a fast steering mirror (FSM) is employed to compensate for tilt errors, and a deformable mirror (DM) is employed to compensate higher order phase distortions. The loop formed by the wavefront sensor, processor and actuator (the conjugating element) imposes a bandwidth constraint on the maximum speed at which pre-distortion can be applied to the outgoing laser beam in the uplink [7, 21, 22]. Hereafter, it is assumed that the wavefront sensor can measure the downlink beacon reference perfectly, and the AO control loop is fast enough to perform the pre-distortion tasks. No further analysis on the PAO bandwidth requirement is performed.

Phase conjugation systems rely on reciprocity, i.e. the outgoing pre-distorted optical wave must travel through the same volume of atmosphere as the sensing beacon, and therefore they work most effectively with a fully shared aperture optical system. In case that the divergence of the output beam needs to be different from the diffraction limited divergence, which is imposed by the diameter of the sensing receiver aperture, zooming optics can be used in the optical path of the outgoing laser beam. In the rest of the analysis it is assumed that the sensing of the downlink is performed within the same telescope used for the uplink transmitter, where the aperture size is set to $20 \mathrm{~cm}$.

There exists a certain degree of decorrelation between the path where the downlink is sensed and the transmission path in the uplink, due to the dichotomy between the PAA and the IPA. In terms of Zernike mode correction, this mismatch translates into a performance reduction that can be approximated by the following expression [22]:

$$
N_{\mathrm{eff}} \approx\left\lfloor N \exp \left\{-\left(\frac{\mathrm{PAA}}{\mathrm{IPA}}\right)^{5 / 3}\right\}\right\rfloor,
$$

where $\lfloor\cdot\rfloor$ is the floor operator, $N$ is the number of Zernike modes that the AO system is trying to correct, and $N_{\text {eff }}$ is the effective number of Zernike modes that AO system can actually correct due to the mismatch between the down and uplink.

Applying pre-distortion $\mathrm{AO}$ correction to an optical wavefront in the uplink, aberrated by atmospheric turbulence, is accomplished by applying the conjugated phase of the reference distorted wavefront. After correction of the first $N_{\text {eff }}$ Zernike modes, the residual phase variance is reduced [9]. This is because there is an inverse relation between the phase variance and the Fried parameter $r_{0}$, whereby $r_{0}$ can be effectively increased as a function of the number $N_{\text {eff }}$ of Zernike 


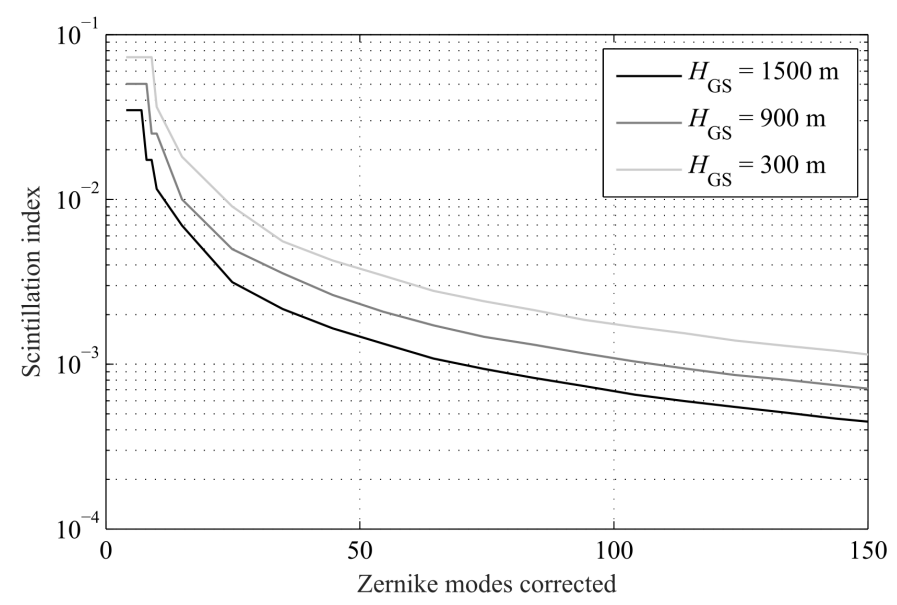

Figure 4. Scintillation index vs. number of Zernike modes corrected including isoplanatic effects for different OGS altitudes at $55^{\circ}$ elevation angle

modes corrected. The expression for a generalized Fried parameter $r_{0, N_{\text {eff }}}$, in terms of the number of Zernike modes corrected, is given by [23]:

$$
r_{0, N_{\text {eff }}}=0.286 r_{0}\left(\frac{3.44}{C_{N}}\right)^{3 / 5} N_{\text {eff }}^{-0.362} ; C_{N}=0.2944 N_{\text {eff }}^{-\sqrt{3} / 2} .
$$

This expression is used hereafter when quantifying the beam wander effects in (5), as well as in the link budget calculation in the next Section 4.

Regarding the possible scintillation index improvement when applying pre-distortion $\mathrm{AO}$ in an optical GEO uplink, Tyson [22] proposes the relation $\sigma_{I, N_{\text {eff }}}^{2}=\sigma_{I}^{2} / N_{\text {eff }}$, where $\sigma_{I, N_{\text {eff }}}^{2}$ is the SI after correction of $N_{\text {eff }}$ Zernike modes, and $\sigma_{I}^{2}$ is the SI without any AO mode correction. This expression results from the fact that $\mathrm{AO}$ correction can be interpreted as a diversity scheme. Figure 4 shows the improvement of SI when $N$ Zernike modes are corrected, including the PAA vs. IPA effect in the analysis.

\section{CHANNEL REALIZATIONS AND LINK BUDGETS}

In this Section, the evaluation scenarios for the study of the performance improvements of a digital transmission scheme performance by the application of pre-distortion adaptive optics technology at the transmitter side in the uplink are defined. OGS altitudes of $300 \mathrm{~m}, 900 \mathrm{~m}$ and $1500 \mathrm{~m}$ are considered for elevation of $55^{\circ}$ as pointed out in Section 2. Two scenarios for the number of wavefront modes to be pre-distorted are considered: 1) pre-distortion of the tilt only, i.e. $N=3$ Zernike modes corrected, and 2) $N=100$ where a large portion of the wavefront errors are corrected. The latter is the required number of Zernike modes to be corrected, in order to achieve about two orders magnitude improvement on the scintillation index as shown in Figure 4.

The dynamic losses of the fading channel in the forward link are modelled by means of channel vectors generated as shown in Figure 1 and countered by the digital coding scheme. In the 
return link, the scintillation is averaged by the large-aperture OGS telescope and limited within a margin [3, 24]. Both in the forward and the return link, optimization of the the beam divergence is performed, equivalent to optimization of the aperture diameter, in order to obtain the lowest possible losses in the optical link. For this purpose, the expression for the average optical power $P_{\mathrm{R}}$ detected at distance $d$ is given as follows:

$$
P_{\mathrm{R}}=P_{\mathrm{T}} G_{\mathrm{T}} \eta_{\mathrm{T}} L_{\mathrm{ATM}} L_{\mathrm{FS}} L_{\mathrm{SR}} L_{\mathrm{BW}} L_{\mathrm{SI}} G_{\mathrm{R}} \eta_{\mathrm{R}}
$$

where $P_{\mathrm{T}}$ is the transmitted average power from a source with aperture diameter $D_{\mathrm{T}}$ which produces a half-angle divergence $\theta_{\mathrm{T}}=\lambda \sqrt{8} / \pi D_{\mathrm{T}} . G_{\mathrm{T}}=\left(\pi D_{\mathrm{T}} / \lambda\right)^{2}$ and $G_{\mathrm{R}}=\left(\pi D_{\mathrm{R}} / \lambda\right)^{2}$ are the transmitter and receiver gains, respectively. $\eta_{\mathrm{T}}$ and $\eta_{\mathrm{R}}$ are the transmitter and receiver efficiency, respectively. $L_{\mathrm{ATM}}$ is the atmospheric attenuation, and $L_{\mathrm{FS}}=(\lambda / 4 \pi d)^{2}$ is the free-space loss. $L_{\mathrm{BW}}=\exp \left(-G_{\mathrm{T}} \theta_{\mathrm{BW}}^{2}\right)$ corresponds to the beam wander loss [25], where $\theta_{\mathrm{BW}}=\sqrt{\left\langle r_{c}^{2}\right\rangle} / d$ is the angular beam wander, and $L_{\mathrm{SR}}=\left[1+\left(D_{\mathrm{T}} / r_{0}\right)^{5 / 3}\right]^{-6 / 5}$ is the Strehl ratio loss which accounts for the broadening of the beam size beyond of that expected due to pure diffraction. It can be readily seen in Figure 5 how an optimum aperture diameter for a given beam divergence, or equivalently an optimum beam divergence for a given transmitter aperture, exists for each of the considered scenarios. In general, aperture size and beam divergence are coupled parameters. However, the application of zooming optics is assumed in this study, which allows for their decoupling and enables independent optimization of the parameter of choice. For example, for accommodation reasons at the OGS, the transmitter aperture may be restricted to a practical size, e.g. $20 \mathrm{~cm}$, and as a result, the beam divergence is to be optimized using a zooming optics system. Such optimization procedure ensures that the losses in the optical GEO feeder link are kept to a minimum. It is shown that the total link loss is decreased by correcting a higher number of Zernike modes, increasing the altitude and increasing the transmitter aperture/divergence. Hereafter, in all calculations where the beam divergence is needed, it is assumed that the optimum divergence value for the respective scenario is used.

In order to calculate the scintillation margin in the return link, the scintillation loss $L_{\mathrm{SI}}$ is defined for a given target availability. Assuming that the PDF of the fading channel can be described with a log-normal distribution, which is in accordance with the channel model presented in 2 , the scintillation loss can be estimated as follows [24]:

$$
L_{\mathrm{SI}}[\mathrm{dB}]=\left(3.3-5.77 \sqrt{\ln \left(\frac{1}{p}\right)}\right) \sigma_{I}^{4 / 5},
$$

where $p$ is the outage probability. Here, $p=0.001$ is assumed for the downlink to ensure $99.9 \%$ availability.

The resulting link budgets, the scintillation indices and the Greenwood frequencies are summarized in Table I. If the cloud coverage on the primary link exceeds the accounted margin for thin cirrus clouds [26], a number of redundant gateways are considered which enable decorrelation of the weather conditions and link handover. For this purpose, a separate set of telescopes can be accommodated in the satellite payload for seamless link acquisition. 

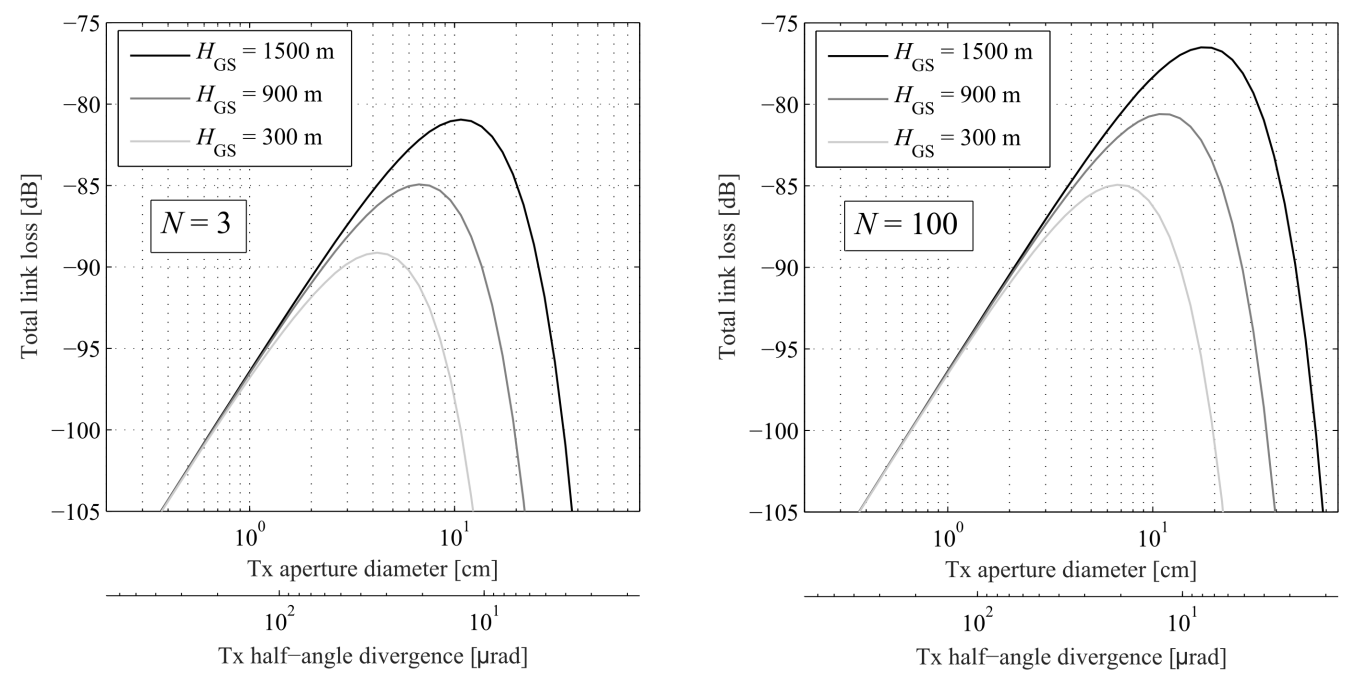

Figure 5. Total uplink loss vs. diffraction limited transmitter size, as well as half-angle divergence, for (left) $N=3$ and (right) $N=100$ Zernike modes corrected, and for different OGS altitudes at $55^{\circ}$ elevation angle.

\begin{tabular}{|c|c|c|c|c|c|c|c|}
\hline Parameter & \multicolumn{6}{|c|}{ Uplink } & Downlink \\
\hline OGS altitude $[\mathrm{m}]$ & 1500 & 900 & 300 & 1500 & 900 & 300 & $\overline{-}$ \\
\hline Modes corrected & \multicolumn{3}{|c|}{$N=3$} & \multicolumn{3}{|c|}{$N=100$} & - \\
\hline Tx aperture diameter $[\mathrm{cm}]$ & \multicolumn{6}{|c|}{20.0} & 25.0 \\
\hline Beam divergence [ $\mu \mathrm{rad}]$ & 12.95 & 20.46 & 33.23 & 7.75 & 12.40 & 20.46 & 5.58 \\
\hline Tx power [W] & \multicolumn{6}{|c|}{50.0} & 1.0 \\
\hline $\mathrm{Rx}$ aperture diameter $[\mathrm{cm}]$ & \multicolumn{6}{|c|}{25.0} & 50.0 \\
\hline Tx power $[\mathrm{dBm}]$ & \multicolumn{6}{|c|}{47.0} & 30.0 \\
\hline Tx gain $[\mathrm{dB}]$ & 106.79 & 102.81 & 98.60 & 111.25 & 107.16 & 102.81 & 114.1 \\
\hline Tx optical loss $[\mathrm{dB}]$ & \multicolumn{7}{|c|}{-3.0} \\
\hline Free-space loss [dB] & \multicolumn{7}{|c|}{-289.50} \\
\hline Atmospheric attenuation $[\mathrm{dB}]$ & \multicolumn{7}{|c|}{$\begin{array}{r}-1.0 \\
30\end{array}$} \\
\hline Cloud margin $[\mathrm{dB}]$ & \multicolumn{7}{|c|}{-3.0} \\
\hline Strehl ratio loss $[\mathrm{dB}]$ & \multicolumn{6}{|c|}{-0.82} & - \\
\hline Beam wander loss $[\mathrm{dB}]$ & \multirow{2}{*}{\multicolumn{6}{|c|}{-4.45}} & - \\
\hline Scintillation margin $[\mathrm{dB}]$ & & & & & & & -1.0 \\
\hline Rx gain $[\mathrm{dB}]$ & \multicolumn{6}{|c|}{114.1} & 120.1 \\
\hline Rx optical loss [dB] & \multicolumn{6}{|c|}{-3.0} & -3.0 \\
\hline Total Rx power $[\mathrm{dBm}]$ & -36.96 & -40.94 & -45.14 & -32.5 & $\mid-36.59$ & -40.94 & -36.3 \\
\hline Scintillation index & 0.0275 & 0.0424 & 0.0625 & 0.0003 & 0.0006 & 0.0012 & 0.002 \\
\hline Greenwood frequency [Hz] & 25.73 & 33.89 & 48.07 & 25.73 & 33.89 & 48.07 & - \\
\hline
\end{tabular}

Table I. Link budget and channel parameters for $55^{\circ}$ elevation in the studied scenarios with pre-distortion adaptive optics applied in the uplink.

The PDFs of the normalized optical channel gains of the fading processes in the forward link are presented in Figure 6 (left). A notable reduction of the variance of the PDFs corresponding to the reduction of the scintillation index can be observed with the increase of the number of wavefront modes to be pre-distorted. From the synthesized channel vectors, the PDFs of the fade durations are computed and presented in Figure 6 (right). It can be confirmed that the fade durations follow 

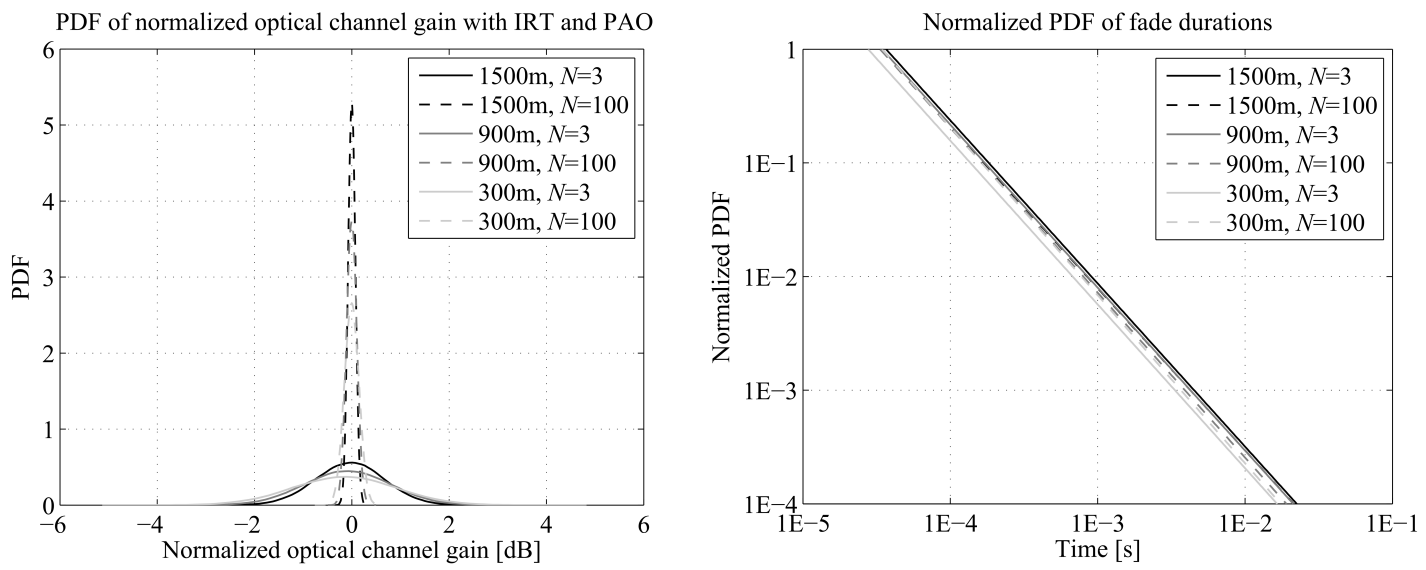

Figure 6. PDF of normalized optical channel gain with IRT (left) and normalized PDF of fade durations (right) with pre-distortion adaptive optics.

an exponential distribution. For the design of a FEC scheme, codewords of $10 \mathrm{~ms}$ are considered to counter the great majority of IRT fades.

\section{DIGITAL TRANSMISSION SCHEME}

In this study, a digital transmission scheme is studied for application over a satellite optical feeder link based on digitization of the RF baseband DVB-S2X signal by means of high-speed analog-to-digital converters (ADCs) and digital-to-analog convertors (DACs) [27], as illustrated in Figure 7. The produced samples are multiplexed together and transmitted by the OGS to the satellite. Assuming a 4-color frequency reuse scheme, the beam bandwidth in the Ka band for the forward link is $1.45 \mathrm{GHz}$ [1]. In the return link, some beams have a bandwidth of $504 \mathrm{MHz}$, while others have $547.5 \mathrm{MHz}$. Assuming a $5 \%$ roll-off factor, 3 carriers of $425 \mathrm{Ms} / \mathrm{s}$ can be accommodated in the forward link. In the return link, 25 carriers of $20.7 \mathrm{Ms} / \mathrm{s}$ fit within the larger piece of bandwidth. Taking into account that there are 150 beams per satellite in the BATS system consisting of two satellites, the resulting traffic on the feeder links is in the order of Terabit/s.

In this paper, a digital transmission scheme for the optical feeder link is proposed. In the forward and the return links, the signal on the feeder link is a digitally modulated optical carrier at $1550 \mathrm{~nm}$. The individual building blocks of the gateway and the satellite are presented, including the optical architecture, the RF-optical interface and the digital processing architecture. In addition, a FEC scheme is developed to cope with the channel fades in the forward link.

\subsection{Forward link}

The digital transmission scheme in the forward link is presented in Figure 8 for one optical terminal of the gateway-satellite chain. As it will be elaborated further on, two terminals would be required, in order to accommodate the required traffic. Each terminal has a transmission and reception chains. Here, half of the data streams per satellite, i.e. 150 beams $/ 2=75$ data streams are assigned to a terminal. 


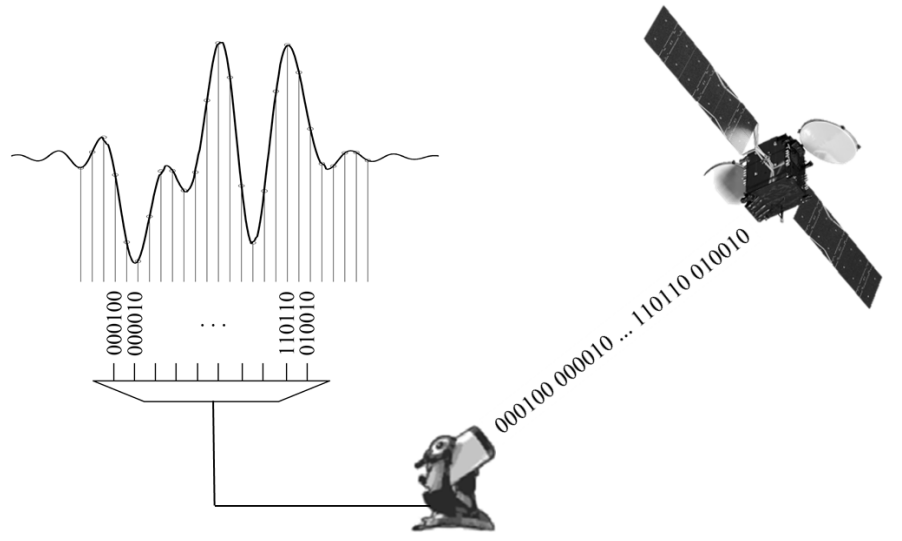

Figure 7. Digital transmission scheme based on digitization of the RF DVB-S2X signal.

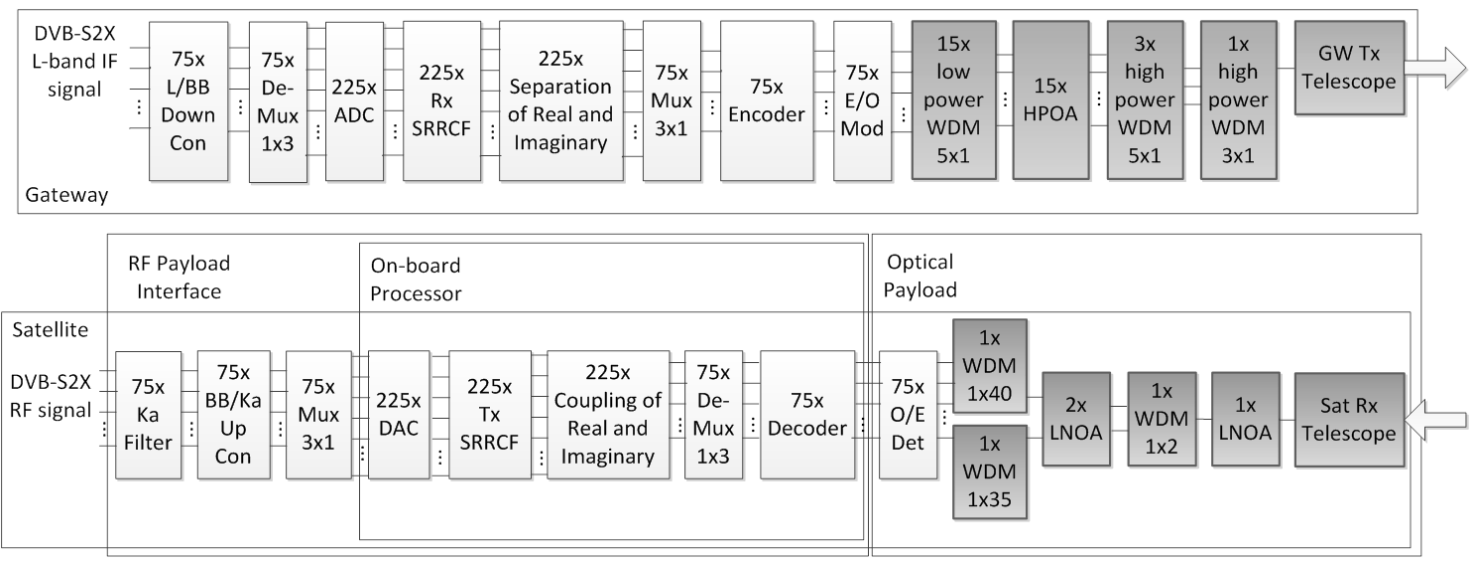

Figure 8 . Block diagram of the digital transmission scheme for the forward optical feeder link.

5.1.1. Processing at the OGS: The DVB-S2X signal typically arrives at the gateway at an intermediate frequency (IF) in the RF L band. The RF-optical interface converts the signal in the desired optical transmission format. After down-conversion to baseband, the $1.45 \mathrm{GHz}$ signal is de-multiplexed to obtain the signals on each of the 3 carriers.

The carrier signals are processed by an ADC to produce a 6-bit digital representation of the real (in-phase) and imaginary (quadrature) signal components. According to the Shannon rate distortion function [28], 6 bits per dimension are sufficient to ensure a signal-to-distortion ratio (SDR) of up to $36 \mathrm{~dB}$. For complex-valued signals with correlated real and imaginary components, such as signals with symbols from amplitude and phase shift keying (APSK) constellations, as well as PSK constellations, commonly used in DVB-S2X, the Linde-Buso-Gray (LBG) algorithm [29] can be used to design a non-uniform vector quantizer with optimum decision regions according to the signal distribution. Given the fact that the APSK constellations in DVB-S2X are optimized with a closeto-Gaussian distribution over the complex plane, the optimum vector quantization of this Gaussian source results in an SDR of approximately $32 \mathrm{~dB}$ with 12 bits per complex-valued symbol. Since the atmospheric optical channel constrains the received signal-to-noise ratio (SNR) to significantly 
lower values, e.g. up to $20.6 \mathrm{~dB}$ as to be presented in Section 5, and 256-APSK on the user link requires at most $24 \mathrm{~dB}$ of SNR [30], the $32 \mathrm{~dB}$ of SDR are considered sufficient to convey the information. However, it has to be noted that the SDR of the quantization on the feeder link is a limiting parameter for the overall SNR on the link. If the evolution of optical technology permits higher SNR in the optical channel, a higher number of bits in the representation of the symbols would be required, should higher constellation orders be used in subsequent standard evolution.

In general, the signal can be up-sampled by a factor of 4 or 8 , in order to maximize the SNR over the APSK information symbols at the subsequent matched filter, a receive square root raised cosine filter (SRRCF). Here, a 6-bit resolution per real and imaginary components on a $425 \mathrm{Ms} / \mathrm{s}$ signal taking into account the above up-sampling factors is considered feasible in an ADC [27]. Nowadays, state-of-the-art ADCs run at Gigasample/s speeds. Instead of interleaving multiple ADC cores to achieve the high speed, often resulting in interleaving artifacts, simplified routing following the high speed serialize/deserialize JESD204B standard and fast overrange detection provide means for direct RF digitization [27]. Even though such components exist for terrestrial applications, they need to pass a space qualification process for the time frame $2020-2025$. Since the signal is to be transmitted over an optical feeder link, where optical and electrical filters are applied for the mapping on the wavelength division multiplexing (WDM) channels preserving the signal integrity, no digital transmit pulse shaping is required. Therefore, the signal can be down-sampled back to the Nyquist rate.

After separation of the real and imaginary components, the bit stream is multiplexed and fed to the FEC encoder to protect the signal from the deep and long fade events in the atmospheric optical channel. The resulting digital bit stream has a rate of $425 \mathrm{Ms} / \mathrm{s} \times 12$ bits per complex-valued sample $\times 3$ carriers $=15.3 \mathrm{~Gb} / \mathrm{s}$ per beam, which after consideration of the subsequently applied coding rate of 0.81 is increased to $18.9 \mathrm{~Gb} / \mathrm{s}$. The structure and processing requirements of the encoder are presented in the next Section 4. Even though encoding at $\mathrm{Gb} / \mathrm{s}$ speeds is shown to be feasible [31], the fact that there are 75 bit streams requires a respective expansion of the hardware and parallelization of the processing possibly on 75 processing units.

The encoded bit stream is passed through the optical architecture, where it is converted into a NRZ-OOK optical signal by means of a Mach-Zender Modulator (MZM). The total of 75 NRZOOK streams are multiplexed on 75 WDM channels. First, 5 channels are bundled together in a $5 \times 1$ low power WDM, and a high-power optical amplifier (HPOA), e.g. an erbium doped fiber amplifier (EDFA), is employed to boost the signal. In total, 15 EDFAs are required. The 15 streams are further multiplexed over a two-stage high-power WDM. Here, three $5 \times 1$ high-power WDMs are first employed, and the output is multiplexed together in one $3 \times 1$ high-power WDM. Finally, the optical signal is transmitted by the gateway transmit telescope.

5.1.2. Processing at the satellite: On board the satellite, the signal is captured by the optical receive telescope as a part of the optical payload. After injection into a single-mode optical fiber, the signal is pre-amplified at a low-noise optical amplifier (LNOA), e.g. an EDFA, and the 75 WDM channels are de-multiplexed and further amplified. A $1 \times 2 \mathrm{WDM}$ splits the streams into two bundles of 40 and 35 channels. Each one of the bundles in amplified at an LNOA. Then, one $1 \times 40 \mathrm{WDM}$ and one $1 \times 35$ WDM de-multiplex the individual channels. The optical signals are optical-electrically 
converted by means of positive-intrinsic-negative (PIN) detectors and passed to the satellite onboard digital processor.

First, each signal is decoded to obtain the received bits. The structure, processing and memory requirements of the decoder are discussed in Section 6. Even though decoding at Gb/s speeds is shown to be feasible [31], the digital on-board processor requires a memory block of estimated 1.3 Gbits per beam (12 Gbytes per terminal), in order to counter the long fading events. After de-multiplexing of the bit stream and coupling of the real and imaginary components, the signal can be up-sampled by a factor of 4 or 8 , before it is passed through a digital pulse shaping filter, a transmit SRRCF, in order to ensure the signal integrity for transmission over the Ka-band user link. Next, the DAC reconstructs the DVB-S2X constellation symbols. The DAC is required to accommodate the dynamic range of the transmitted constellation. In the forward link, the normalized APSK constellation of the DVB-S2X standard has an order of up to 256. This results in a maximum variation of the power of the symbols (i.e. the ratio of largest symbol power to smallest symbol power) at the output of the DAC of up to $20 \mathrm{~dB}$ for 256-APSK. The two error sources considered in this study, the fading channel and the additive white Gaussian noise (AWGN) at the receiver, can flip some bits of the digital signal at the input of the DAC. The signal after the DAC can be considered noise-free due to the performed detection of the NRZ-OOK stream, but not error-free, i.e. some quantized levels other than the ones of the signal constellation may appear due to the flipped bits. However, with a proper design of the FEC code, the probability of such error events can be minimized.

After multiplexing of 3 analog carriers per beam, the obtained baseband user beam signal is upconverted to Ka band. Here, and integrated two-stage solution can be considered, i.e. baseband to $\mathrm{C}$ band and $\mathrm{C}$ band to Ka band, to ensure an efficient up-conversion process. After filtering through a Ka band channel filter, the signal is passed to the RF front-end and the satellite antenna, so it can be transmitted over the forward user downlink.

\subsection{Return link}

The transmission chain for the return link is presented in Figure 9. As explained in the previous section, the block diagram of the receive chain of a single optical terminal is presented, while two terminals in total are required. The major difference with the forward optical feeder link is that the receiver at the gateway employs a large aperture telescope in the order of $50 \mathrm{~cm}$ which averages the scintillation within a margin and successfully counters the fades in the atmospheric optical channel. Therefore, no FEC is required to protect the optical signal on the return feeder link.

5.2.1. Processing at the satellite: After reception of the DVB-RCS2 signal at the antenna and RF payload interface, it is down-converted to baseband, and the 25 carriers in the $547.5 \mathrm{MHz}$ signal are de-multiplexed. Here, again a two-stage down-conversion process, i.e. Ka band to $\mathrm{C}$ band and $\mathrm{C}$ band to baseband, can be employed to increase the conversion efficiency.

The ADC in the on-board processor samples each carrier with a 6-bit resolution per real and imaginary component. In general, the DVB-RCS2 carriers are modulated by means of quadrature phase shift keying (QPSK), 8-PSK, and 16-level quadrature amplitude modulation (16-QAM). The signal can be up-sampled by a factor of 4 or 8 to maximize the SNR over the symbols at the following matched filter, a receive SRRCF. In the return link on board the satellite, the 6-bit resolution per real 

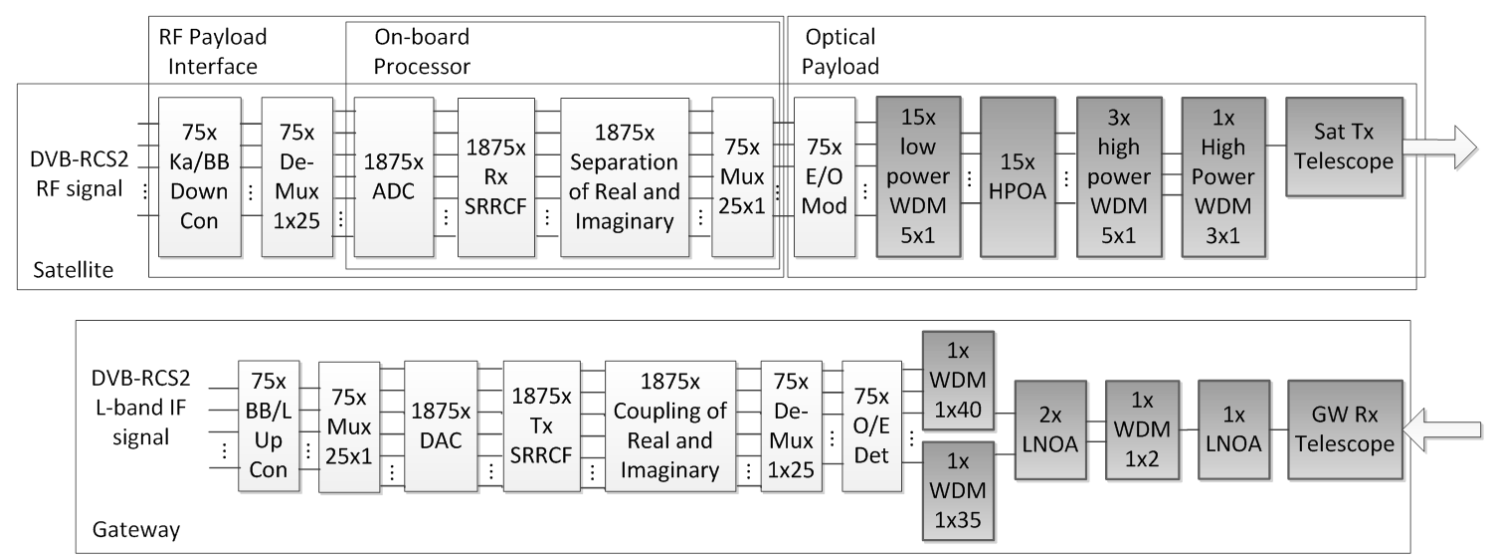

Figure 9. Block diagram of the digital transmission scheme for the return optical feeder link.

and imaginary components on a $20.7 \mathrm{Ms} / \mathrm{s}$ signal taking into account the above up-sampling factors is much less of a challenge for an ADC [27]. Since no digital transmit pulse shaping is required for transmission over the optical feeder downlink, the signal can be down-sampled back to the Nyquist rate. The digitized real and imaginary components are multiplexed into a bit stream with a rate of $20.7 \mathrm{Ms} / \mathrm{s} \times 12$ per complex-valued sample $\times 25$ carriers $=6.2 \mathrm{~Gb} / \mathrm{s}$ per beam.

In the optical payload, the bit stream is modulated by a MZM into a NRZ-OOK optical signal. The total of 75 NRZ-OOK streams are multiplexed on 75 WDM channels. Similarly, 5 channels are bundled together in a $5 \times 1$ low power WDM, and they are amplified by a high-power EDFA, requiring a total of 15 EDFAs. The 15 streams are further multiplexed over a two-stage high-power WDM. Here, three $5 \times 1$ high-power WDMs are first employed, and the output is multiplexed together in one $3 \times 1$ high-power WDM. Finally, the optical signal is transmitted by the satellite transmit telescope.

5.2.2. Processing at the OGS: At the OGS, the signal is captured by the optical receive telescope. After injection into a single-mode optical fiber, the signal is pre-amplified at an LNOA, and the 75 channels are split into two bundles of 40 and 35 channels at a $1 \times 2$ WDM. Each one of the bundles in amplified at an LNOA. Then, one $1 \times 40 \mathrm{WDM}$ and one $1 \times 35 \mathrm{WDM}$ de-multiplex the individual channels. The optical signals are optical-electrically converted by means of PIN detectors.

After de-multiplexing of the bit stream and coupling of the real and imaginary components, the signal can be up-sampled by a factor of 4 or 8 , before it is passed through a digital pulse shaping filter, a transmit SRRCF, in order to ensure compatibility with the processing at the hub. Next, the DAC reconstructs the DVB-RCS2 constellation symbols. In the return link, the maximum 16-QAM constellation requires a DAC dynamic range up to $9.5 \mathrm{~dB}$. After multiplexing of 25 analog carriers, the obtained baseband signal per beam can be up-converted to an intermediate frequency in the RF $\mathrm{L}$ band, and it is transported to the hub. 


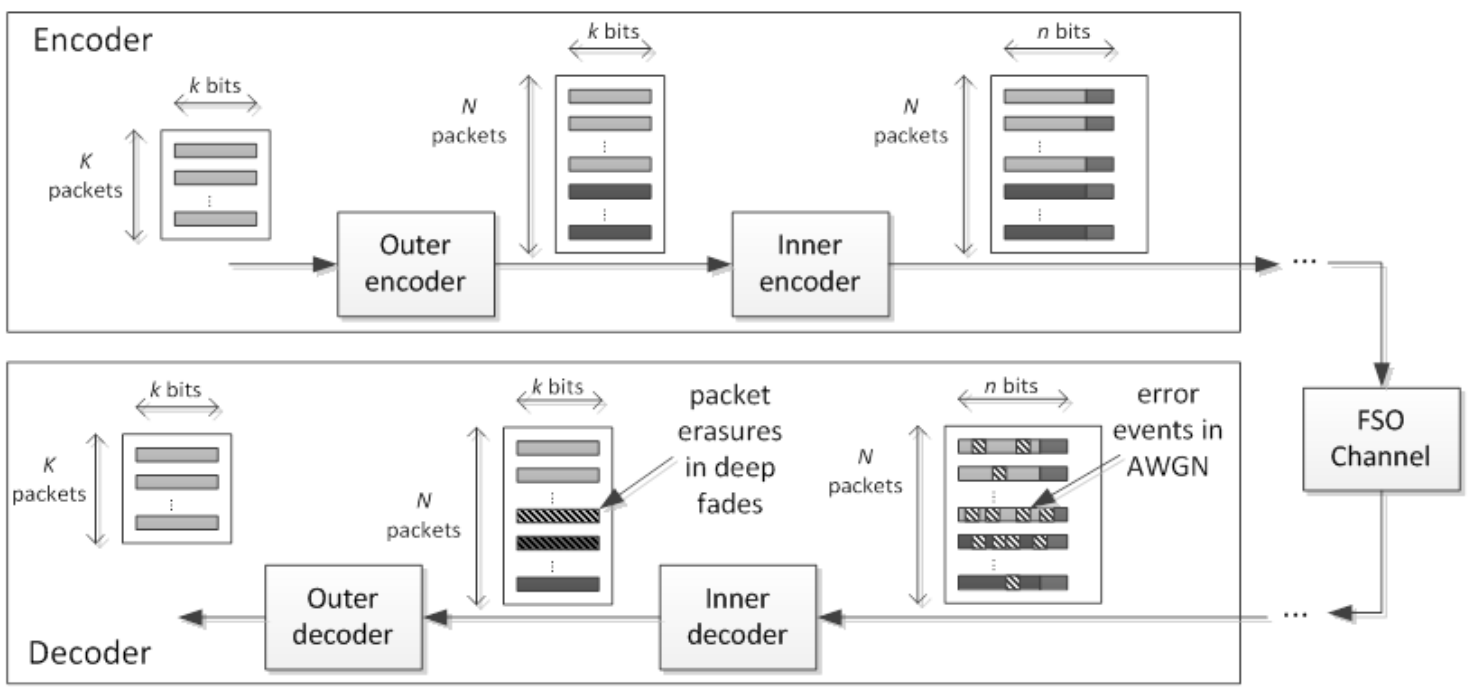

Figure 10. Block diagram of the encoder and decoder for the forward optical feeder link.

\section{PACKET-LEVEL CODING}

The correlated fading events due to IRT pose a significant challenge to the transmission on the feeder link, especially on the forward link. The deep and long fading events over the optical link due to IRT render the inclusion of a fade mitigation margin impractical for the link budget. In addition, the adaptive coding and modulation (ACM) scheme of DVB-S2X can only compensate for slow fading due to rain, as well as for the difference in the user channel gains due to the antenna pattern on the user link. Therefore, a suitable FEC scheme with an appropriate framing structure is required. In this Section, a low-complexity FEC scheme is proposed for the digital signal on the forward optical feeder link, tailored to the atmospheric channel characteristics and the following assumptions: (1) data rate in the order of $20 \mathrm{~Gb} / \mathrm{s}$ as discussed in Section 5, (2) fade durations in the order of $10 \mathrm{~ms}$ as presented in Section 4 and (3) a target packet-error rate (PER) of less than $10^{-7}$, in order to meet the end-to-end Quality of Service (QoS) of DVB-S2X. The designed FEC scheme in Figure 10 is composed of two parts:

- a low-complexity inner code, necessary for coping with random sporadic errors due to AWGN;

- a low-complexity long erasure correcting code, also referred to as outer code, which takes care of recovering lost packets due to deep fade events.

Additionally, after the inner decoder, an error detection scheme is required either in form of a CRC or inherently as part of the inner code. Initially, a set of $K$ packets, made of $k$ bits each, is buffered at the gateway. The outer encoder computes $N-K$ additional redundancy packets at a code rate of $R=K / N$. Each of the $N$ output packets is protected with an inner code. More specifically, the inner encoder appends to each packet $n-k$ parity bits at a code rate of $r=k / n$. The channel introduces sporadic errors due to AWGN, and long bursty errors due to fades. The inner decoder tries to correct the errors in each packet. If the number of errors in a packet does not exceed the error 
correction capability of the code, the packet is correctly recovered. Otherwise, the packet is marked as erased. Note that an undetected error may occur, if the decoder produces a decision which is a valid codeword different from the transmitted one. The probability of this event can be kept small by adopting suitably designed codes with incomplete decoding algorithms. Subsequently, the outer decoder attempts to recover the erased packets.

For the inner code, a good choice is a low-complexity error correcting code with a high coding rate. Considering the high data rate in the order of $\mathrm{Gb} / \mathrm{s}$ and the duration of the fade events, a $(8191, k)$ Bose-Ray-Chaudhuri-Hocquenghem $(\mathrm{BCH})$ code is a viable option. A possible solution is to adopt a $r=0.95$ shortened $(8000,7610) \mathrm{BCH}$ code with error correction capability of 30 errors, which is not entirely used. In particular, the error correction capability can be limited to 29 errors (or less, if required) to allow for sufficiently low undetected error probabilities [32]. The duration of each codeword is $T_{\mathrm{CW}}=8191 /\left(20 \times 10^{9}\right)=4 \times 10^{-7}$ seconds. In a fade event of $10 \mathrm{~ms}, 25000$ codewords are lost. For a target outer code rate of $R=0.85$, the block length needs to exceed $25000 /(1-R)=160000$ packets. An idealized maximum distance separable erasure correcting code, e.g. a Reed-Solomon code, can recover up to $n \times(1-R)$ erased symbols. However, such codes do not exist for an arbitrary set of parameters such as block length, code rate and Galois field order. Moreover, their decoding complexity does not scale favorably with the block length, leading to impractical decoding complexities for large blocks. Sparse-graph-based erasure correcting codes, e.g. LDPC codes, are known to attain the performance bound for large block lengths under iterative decoding [12]. Erasure decoding of such codes has been demonstrated at speeds of several $\mathrm{Gb} / \mathrm{s}$ [31].

According to these design rules, the receiver on-board unit can perform hard-decision boundeddistance decoding of the $\mathrm{BCH}$ code, e.g. via the Berlekamp-Massey algorithm. If this step is too complex, the BCH code can be replaced by a LDPC code with same block length and code rate, where bit-flipping decoding is performed. In addition, the receiver also performs erasure decoding with the long LDPC code, where the complexity can be kept remarkably small [31]. The proposed approach has a memory requirement to store the $N \times k$ bits on board for decoding. With $N=160000$ and $n=8191$, on-board memory of approximately $1.3 \mathrm{Gbits}=162.5$ Mbytes per beam is required. This requirement cannot be removed by any fully regenerative scheme due to the amount of bits involved in a $10 \mathrm{~ms}$ fade (200 Mbits) and the high overall code rate of 0.81 . In a system with e.g. 75 beams per optical terminal, a total memory size of approximately 12 GBytes is required. Implementation of the digital transmission scheme is envisioned on field programmable gate arrays (FPGAs), whereby the DAC and ADC can be included as part of the FPGA board. The digital signal processing and memory requirements of the on-board terminal are summarized in Table II.

\section{PERFORMANCE EVALUATION}

The performance of the digital transmission scheme is assessed in the presence of the receiver noise and the fading in the optical wireless channel. First, the optical bandwidth requirements to support the Terabit/s traffic are considered. The bit rates per beam in the forward link amount to $15.3 \mathrm{~Gb} / \mathrm{s}$ and $18.9 \mathrm{~Gb} / \mathrm{s}$ for the uncoded and coded schemes, respectively, while $6.2 \mathrm{~Gb} / \mathrm{s}$ per beam are required in the return link. One or several beams can be allocated to a WDM channel. However, since the designed FEC scheme is applied to the individual streams, it is preferable that 


\begin{tabular}{|c|c|c|c|c|}
\hline \multirow{2}{*}{$\begin{array}{l}\text { Digital on-board } \\
\text { processor }\end{array}$} & \multicolumn{2}{|r|}{ Uplink } & \multicolumn{2}{|r|}{ Downlink } \\
\hline & $\begin{array}{l}\text { FPGA } \\
\text { (performs } \\
\text { decoding) }\end{array}$ & DAC & FPGA & $\mathrm{ADC}$ \\
\hline $\begin{array}{l}\text { Device count per } \\
\text { subsystem }\end{array}$ & 75 & 225 & 75 & 1875 \\
\hline $\begin{array}{l}\text { Signal } \\
\text { processing } \\
\text { speed }\end{array}$ & $18.9 \mathrm{~Gb} / \mathrm{s}$ & $\begin{array}{l}425 \mathrm{Ms} / \mathrm{s} \text { at } 12 \text { bits per } \\
\text { complex-valued sym- } \\
\text { bol with } 4 \text { or } 8 \text { over- } \\
\text { sampling }\end{array}$ & $6.2 \mathrm{~Gb} / \mathrm{s}$ & $\begin{array}{l}20.7 \mathrm{Ms} / \mathrm{s} \text { at } 12 \text { bits per } \\
\text { complex-valued sym- } \\
\text { bol with } 4 \text { or } 8 \text { over- } \\
\text { sampling }\end{array}$ \\
\hline $\begin{array}{l}\text { Memory require- } \\
\text { ment }\end{array}$ & $\begin{array}{l}162.5 \\
\text { MBytes }\end{array}$ & - & - & - \\
\hline
\end{tabular}

Table II. Digital signal processing and memory requirements per on-board optical terminal.

\begin{tabular}{|c|c|c|c|c|c|c|c|}
\hline Parameter & \multicolumn{6}{|c|}{ Uplink } & Downlink \\
\hline OGS altitude [m] & 1500 & 900 & 300 & 1500 & 900 & 300 & - \\
\hline Modes corrected & \multicolumn{3}{|c|}{$N=3$} & \multicolumn{3}{|c|}{$N=100$} & - \\
\hline $\begin{array}{l}\text { El. SNR of uncoded } \\
\text { NRZ-OOK, } 15.3 \mathrm{~Gb} / \mathrm{s}[\mathrm{dB}]\end{array}$ & 15.8 & 11.3 & 6.1 & 20.6 & 16.2 & 11.3 & - \\
\hline $\begin{array}{l}\text { El. SNR of coded } \\
\text { NRZ-OOK, } 18.9 \mathrm{~Gb} / \mathrm{s}[\mathrm{dB}]\end{array}$ & 14.9 & 10.4 & 5.2 & 19.7 & 15.3 & 10.4 & - \\
\hline $\begin{array}{l}\text { El. SNR of uncoded } \\
\text { NRZ-OOK, } 6.2 \mathrm{~Gb} / \mathrm{s}[\mathrm{dB}]\end{array}$ & \multicolumn{6}{|c|}{ - } & 20.7 \\
\hline
\end{tabular}

Table III. Available electrical SNRs in the studied scenarios.

the transmission rate is kept as low as possible, in order to alleviate the processing complexity and memory requirements. Therefore, one beam is allocated per WDM channel, resulting in a total of 150 WDM channels. The above mentioned data rates on the forward and return links fit within $50 \mathrm{GHz}(0.4 \mathrm{~nm})$ and $25 \mathrm{GHz}(0.2 \mathrm{~nm})$ WDM grid spacings [33], respectively. In the forward link, a total optical bandwidth of $150 \times 0.4 \mathrm{~nm}=60 \mathrm{~nm}$. In the optical $\mathrm{C}$ and $\mathrm{L}$ bands, the available bandwidth is $30 \mathrm{~nm}$ in each band, resulting in a total of $60 \mathrm{~nm}$ of available optical spectrum. Therefore, the forward link would require the occupation of both optical $\mathrm{C}$ and $\mathrm{L}$ bands. In the return link, the optical bandwidth is $150 \times 0.2 \mathrm{~nm}=30 \mathrm{~nm}$, which can be allocated in the $\mathrm{C}$ or $\mathrm{L}$ band. Since a total of $90 \mathrm{~nm}$ of optical bandwidth is required, two optical terminals are employed, where each terminal handles $30 \mathrm{~nm}$ in the forward link (e.g. in L band) and $15 \mathrm{~nm}$ in the return link (e.g. in $\mathrm{C}$ band), for a total of $45 \mathrm{~nm}$ bandwidth per terminal. As a result, 4 feeder links per OGS site ( 2 feeder links per satellite) are able to accommodate the entire Terabit/s traffic in the BATS system.

The noise at the detector can be modelled as a combination of amplified spontaneous emission (ASE) noise, thermal noise and shot noise [34]. Here, an EDFA gain of $35 \mathrm{~dB}$ is considered with a noise figure of $4 \mathrm{~dB}$, while detector responsivity of $0.9 \mathrm{~A} / \mathrm{W}$ is assumed with a quantum efficiency of 0.72 . The available electrical SNR for a received optical power level is presented in Table III, considering the WDM channel rates.

Using the generated channel vectors, the bit-error rate (BER) performance of uncoded NRZ-OOK can be evaluated in the IRT fading channel. Since a square law detector, i.e. a PIN photodiode, is 


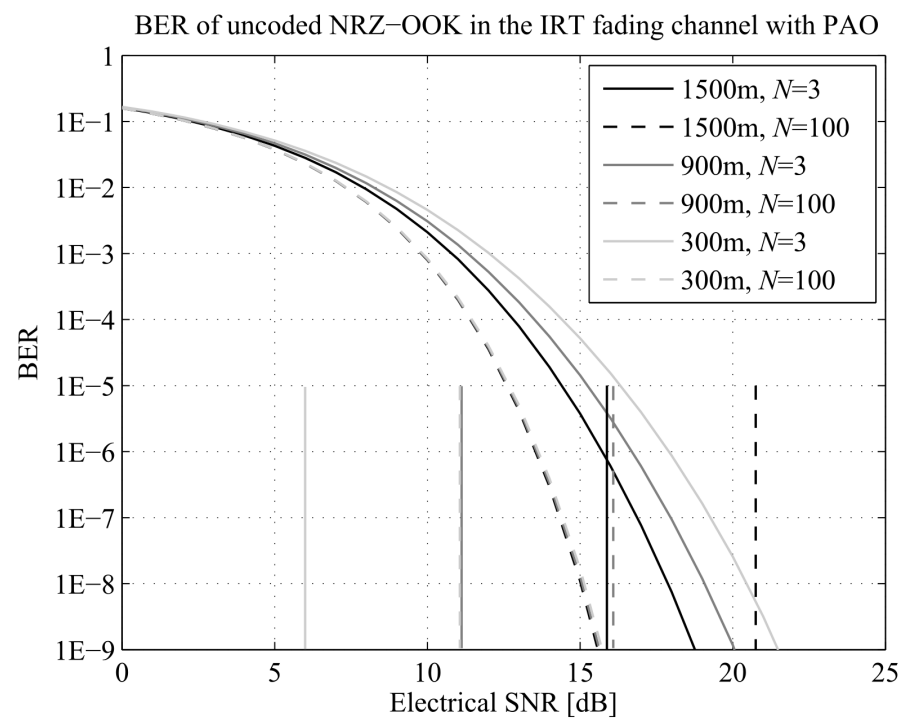

Figure 11. BER of uncoded NRZ-OOK in the forward link, including the available electrical SNR.

applied at the receiver [35], the instantaneous electrical SNR, $\bar{\gamma}$, can be expressed as a function of the instantaneous optical channel gain, $h$, and the average electrical SNR in AWGN, $\gamma$, as follows: $\bar{\gamma}=h^{2} \gamma$. Therefore, the instantaneous electrical SNR is a random variable in the fading channel, and the BER performance of the modulation scheme needs to be averaged over the realisations of the instantaneous SNR [36]. Given the well-known expression for the BER performance of NRZOOK in AWGN [37], the average BER of uncoded NRZ-OOK can be expressed in the IRT fading channel as follows:

$$
\operatorname{BER}_{\substack{\mathrm{NRZ}-\mathrm{OOK} \\ \text { in IRT }}}=\mathrm{E}\left[\operatorname{BER}_{\substack{\mathrm{NRZ}-\mathrm{OOK} \\ \text { in AWGN }}}(\bar{\gamma})\right]=\mathrm{E}\left[\frac{1}{2} \operatorname{erfc}\left(\sqrt{\frac{h^{2} \gamma}{2}}\right)\right],
$$

where $\mathrm{E}[\cdot]$ is the expectation operator.

The BER of uncoded NRZ-OOK in the different fading channels is presented in Figure 11. The available electrical SNR is depicted by the vertical lines. The relationship between the BER and the PER can be established for a packet length of 8191 bits as follows: PER $=1-(1-\mathrm{BER})^{8191}$. Thus, a PER of $10^{-7}$ corresponds to $10^{-9}$ BER. It is shown that the link budget is closed for elevation angle of $55^{\circ}$ at $900 \mathrm{~m}$ and $1500 \mathrm{~m}$ altitudes only when a large portion of the modes are compensated. Here, the target BER of $10^{-9}$ is barely closed at $900 \mathrm{~m}$, while $5.1-\mathrm{dB}$ margin is available at $1500 \mathrm{~m}$.

The PER performance of the packet-level coded NRZ-OOK scheme from Section 3 is evaluated in the different channels by means of Monte Carlo simulations. For this purpose, the generated channel samples are used to compute the decoding error probability at the output of the inner decoder. Mapping each inner codeword onto an outer code packet, the decoding error probability of the inner code serves as input for a virtual packet erasure channel over which the outer code is applied. The results for the PER are presented in Figure 12. The available electrical SNR is depicted by the vertical lines. It is shown that the link budget is closed for elevation angle of $55^{\circ}$ at $1500 \mathrm{~m}$ altitude 


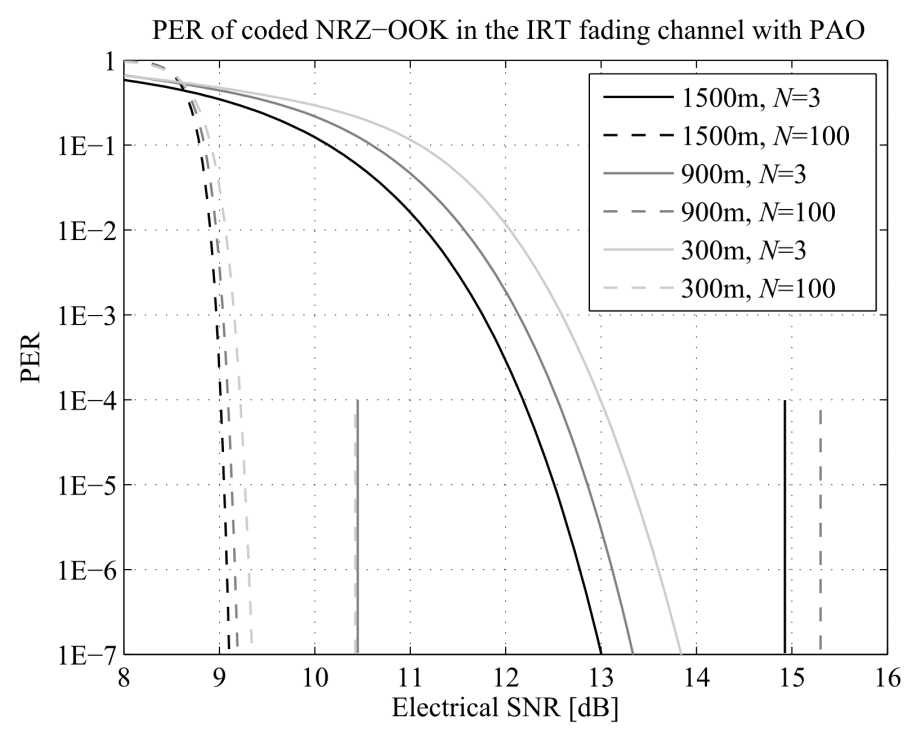

Figure 12. PER of coded NRZ-OOK in the forward link, including the available electrical SNR.

with first 3 modes compensated, while all the scenarios close the link budget when a large portion of the modes are compensated. Here, a 1.92-dB margin is available for $N=3$ at $1500 \mathrm{~m}$. For $N=100$, the available margins amount to $10.6,6.1$ and $1.1 \mathrm{~dB}$ at 1500,900 and $300 \mathrm{~m}$, respectively.

In the return link, the PER requirement is relaxed to $10^{-5}$ [14], which corresponds to a BER of $10^{-7}$. Here, due to scintillation averaging at the $50 \mathrm{~cm}$ large-aperture OGS receive telescope, the scintillation effect can be contained within a small margin as shown in Table I. The received optical power of $-36.3 \mathrm{dBm}$ yields an available electrical SNR of $20.7 \mathrm{~dB}$. Since uncoded OOK in AWGN requires an electrical SNR of $14.5 \mathrm{~dB}$ at $10^{-7} \mathrm{BER}$, it is able to close the link budget with a significant margin of $6.2 \mathrm{~dB}$. Therefore, the digital transmission scheme is shown to successfully meet the feeder link and end-to-end QoS requirements.

\section{CONCLUSION}

In this paper, a digital transmission scheme based on NRZ-OOK is proposed for Terabit/s satellite optical feeder links. Packet-level FEC coding and pre-distortion adaptive optics techniques are proposed as countermeasures of the IRT in the atmospheric optical channel in the forward link. Channel vectors have been generated for a $55^{\circ}$ elevation scenario with OGS altitudes of $300 \mathrm{~m}$, $900 \mathrm{~m}$ and $1500 \mathrm{~m}$. It is shown that pre-distortion adaptive optics with a large number of precompensated modes enables the application of uncoded NRZ-OOK at altitudes of $900 \mathrm{~m}$ and $1500 \mathrm{~m}$ with a margin of $5.1 \mathrm{~dB}$ in the latter case. The addition of packet-level coding enables NRZ-OOK with pre-distortion adaptive optics to close the link budget at $300 \mathrm{~m}, 900 \mathrm{~m}$ and $1500 \mathrm{~m}$ OGS altitude for a large number of pre-compensated modes with margins of $1.1 \mathrm{~dB}, 6.1 \mathrm{~dB}$ and $10.6 \mathrm{~dB}$, respectively. In the case at $1500 \mathrm{~m}$ with only tilt pre-compensation and packet-level coding, the budget is also closed with a margin of $1.9 \mathrm{~dB}$. The study has shown the reduction of the total link loss and the scintillation index by means of pre-distortion adaptive optics which improves the 
performance of both the uncoded and coded NRZ-OOK digital transmission schemes in the forward link. Due to the employed large-aperture OGS receive telescope, the return link successfully closes the link budget even without FEC with a margin of $6.2 \mathrm{~dB}$.

The optical feeder link technology is shown to enable the transmission of the entire feeder link traffic through a single OGS. Considering the number of redundant ground stations to fulfill the required feeder link availability, taking into account the weather conditions, the number of gateways is significantly reduced as compared to an RF feeder solution. As a result, the cost of the ground segment reduced at the expense of the added complexity to the space segment, as well as the ground segment. Even though the electrical-to-optical conversion efficiency of digital NRZOOK transmission may be considered low due to the large optical bandwidth utilization for an effective RF throughput because of the large quantization factor, the studied scheme is shown to be a functioning solution for the forward and return optical feeder links. If the increased complexity of the onboard terminal and the ground station can be accommodated, digital NRZ-OOK transmission with packet-level FEC and pre-distortion adaptive optics in the forward link is shown to be an effective countermeasure of the IRT in the atmospheric optical channel and a viable option for Terabit/s HTS optical feeder links. Further developments will focus on transmission techniques that permit the increase of the bandwidth efficiency, as well as provide fade mitigation in the optical channel, in order to allow for efficient full utilization of the optical feeder link bandwidth.

\section{ACKNOWLEDGEMENT}

This work has been supported by the BATS research project which is funded by the European Union Seventh Framework Programme under contract n317533.

\section{REFERENCES}

1. Broadband Access via Integrated Terrestrial and Satellite Systems (BATS). ICT-2011.1.1 BATS D4.1: Satellite Network Mission Requirements. Technical Report, European Project 2012.

2. Cowley W, Giggenbach D, Mata Calvo R. Optical Transmission Schemes for GEO Feeder Links. Proc. of IEEE ICC 2014, Sydney, Australia, 2014.

3. Andrews LC, Philips RL. Laser Beam Propagation through Random Media. 2nd edn., SPIE Press: Belligham, 2005.

4. Henniger H. Transmission performance analysis of free-space optical communications using gilbert-erasure channel. IEEE Trans. Commun. Jan 2012; 60(1):55 -61.

5. Toyoshima M, Yamakawa S, Yamawaki T, Arai K, Garca-Talavera MR, Alonso A, Sodnik Z, Demelenne B. Longterm statistics of laser beam propagation in an optical ground-to-geostationary satellite communications link. IEEE Trans. Antennas Propag. 2005; 53(2):842-850.

6. Tatarski VI. The effects of the turbulent atmosphere on wave propagation. Israel Program for Scientific Translation, 1971.

7. Tyler GA. Bandwidth considerations for tracking through turbulence. J. Opt. Soc. Am. A 1994; 11(1):358-367.

8. Hardy JW. Active optics: a new technology for the control of light. Proc. IEEE 1977; .

9. Noll RJ. Zernike polynomials and atmospheric turbulence. J. Opt. Soc. Am. 1976; 66(3):207-211.

10. Hayee MI, Willner AE. NRZ versus RZ in 10-40-Gb/s Dispersion-Managed WDM Transmission Systems. IEEE Photon. Technol. Lett. Aug 1999; 11(8):991-993.

11. Kim H, Gnauck AH. Experimental Investigation of the Performance Limitation of DPSK Systems due to non-linear Phase Noise. IEEE Photon. Technol. Lett. Feb 2003; 15(2):320-322.

12. Liva G, Paolini E, Chiani M. Bounds on the Error Probability of Block Codes over the q-Ary Erasure Channel. IEEE Trans. Commun. Jun 2013; 61(2):2156-2165. 
13. Second generation framing structure, channel coding and modulation systems for broadcasting, interactive services, news gathering and other broadband satellite applications; part ii: S2-extensions (dvb-s2x) - (optional) Mar 2014.

14. Second generation dvb interactive satellite system (dvb-rcs2); part 2: Lower layers for satellite standard Jan 2012.

15. Giggenbach D. Optimierung der optischen freiraumkommunikation durch die turbulente atmosphre - focal array receiver. PhD Thesis, Universitët der Bundeswehr München 2004.

16. International Telecommunication Union (ITU); Recommendation ITU-R P1853. Tropospheric Attenuation Time Series Synthesis 2009.

17. Fried DL. Anisoplanatism in adaptive optics. J. Opt. Soc. Am. 1982; 72(1):52-61.

18. Prata AJ, Rusch WVT. Algorithm for computation of zernike polynomials expansion coefficients. Appl. Opt. 1989; 28(4):749-754.

19. Gureyev TE, Roberts A, Nugent KA. Phase retrieval with the transport-of-intensity equation: matrix solution with use of zernike polynomials. J. Opt. Soc. Am. A 1995; 12(9):1932-1941.

20. Dai G. Modal wave-front reconstruction with zernike polynomials and karhunenlove functions. J. Opt. Soc. Am. A 1996; 13(6): 1218-1225.

21. Greenwood DP. Bandwidth specification for adaptive optics systems. J. Opt. Soc. Am. 1977; 6(3):390-393.

22. Tyson RK. Adaptive optics and ground-to-space laser communications. Appl. Opt. 1996; 35(19):3640-3646.

23. Cagigal MP, Canales VF. Generalized Fried parameter after adaptive optics partial wave-front compensation. $J$. Opt. Soc. Am. A 2000; 17(5):903-909.

24. Giggenbach D, Henniger H. Fading-loss assessment in atmospheric freespace optical communication links with on-off keying. Opt. Eng. 2008; 47(4):046001.

25. Arnon S, Kopeika NS, Kedar D, Zilberman A, Arbel D, Livne A, Guelman M, Orenstain M, Ginati HMA. Performance limitation of laser satellite communication due to vibrations and atmospheric turbulence: down-link scenario. Int. J. Satell. Commun. Network 2003; 21:561-573.

26. Churnside JH, Shaik K. Atmospheric Propagation Issues Relevant to Optical Communications. National Oceanic and Atmospheric Administration: Boulder, CO, USA, 1989.

27. Beavers I. Gigasample ADCs Run Fast to Solve New Challenges. Technical Report MS-2702, Analog Devices 2014.

28. Shannon CE. A Mathematical Theory of Communications. The Bell System Technical Journal Jul 1948; 27:379423.

29. Linde Y, Buzo A, Gray RM. An Algorithm for Vector Quantizer Design. IEEE Trans. Commun. Jan 1980; 28(1):8495.

30. Implementation guidelines for the second generation system for broadcasting, interactive services, news gathering and other broadband satellite applications; part 2 - s2 extensions (dvb-s2x) Mar 2015.

31. Liva G, Matuz B, Paolini E, Chiani M. Pivoting Algorithms for Maximum Likelihood Decoding of LDPC Codes over Erasure Channels . Proc. of IEEE GLOBECOM 2009, Honolulu, HI, USA, 2009; 1-6.

32. Kim MG, Lee JH. Undetected Error Probabilities of Binary Primitive BCH Codes for Both Error Correction and Detection. IEEE Trans. Commun. May 1996; 44(5):575-580.

33. International Telecommunication Union (ITU); Recommendation ITU-R G6941. Spectral Grids for WDM Applications: DWDM Frequency Grid 2012.

34. Personick SD. Receiver Design for Digital Fibre Optic Communication Systems, I and II. The Bell System Technical Journal Jul-Aug 1973; 52(6):843-886.

35. Majumdar AK, Ricklin JC. Free-Space Laser Communications: Principles and Advances. Springer, 2008.

36. Simon MK, Alouini MS. Digital Communication over Fading Channels: A Unified Approach to Performance Analysis. John Wiley and Sons, 2005.

37. Li J, Liu JQ, Tayler DP. Optical Communication Using Subcarrier PSK Intensity Modulation Through Atmospheric Turbulence Channels. IEEE Transactions on Communications Aug 2007; 55(8):1598-1606. 